\title{
High expression of long non-coding HOTAIR correlated with hepatocarcinogenesis and metastasis
}

\author{
DA-NI ZHONG ${ }^{1 *}$, YI-HUAN LUO ${ }^{2 *}$, WEI-JIA MO ${ }^{3}$, XIN ZHANG $^{2}$, ZHONG TAN $^{2}$,

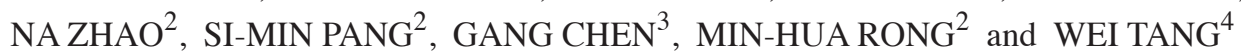 \\ ${ }^{1}$ Department of Chemotherapy; ${ }^{2}$ Research Department, Affiliated Cancer Hospital, Guangxi Medical University; \\ ${ }^{3}$ Department of Pathology, First Affiliated Hospital of Guangxi Medical University; ${ }^{4}$ Department of Breast Surgery, \\ Affiliated Cancer Hospital, Guangxi Medical University, Nanning, Guangxi Zhuang Autonomous Region 530021, P.R. China
}

Received February 25, 2017; Accepted August 14, 2017

DOI: $10.3892 / \mathrm{mmr} .2017 .7999$

\begin{abstract}
HOX transcript antisense RNA (HOTAIR), a newly discovered long noncoding RNA (IncRNA), has been reported to be a poor prognostic marker in many types of cancers. The current study attempted to investigate the biological roles and clinicopathlogical implications of HOTAIR in hepatocellular carcinoma (HCC), as well as understand the molecular mechanisms of HOTAIR in HCC progression. HOTAIR expression in 95 HCC patients with paired HCC tissues and adjacent non-cancer tissues were investigated using quantitative reverse transcription-polymerase chain reaction. The association between HOTAIR expression and clinicopathological features was assessed. The effects of HOTAIR were examined in vitro assays by silencing the IncRNA. Pathway analyses were performed to illustrate the biological functions of the HOTAIR and coexpression genes. The expression level of HOTAIR was observed significantly higher in the HCC tissue than the adjacent non-tumor tissue. HOTAIR expression levels were significantly higher in tumor samples from patients with distant metastasis, advanced stage, portal vein tumor embolus, vasoinvasion, tumor capsular infiltration or positive nm23 expression than those from patients without these conditions, correspondingly. The silencing of HOTAIR in liver cancer cells induced the inhibition of cell proliferation and promotion of apoptosis. Several pathways such as extracellular matrix-receptor
\end{abstract}

Correspondence to: Dr Min-Hua Rong, Research Department, Affiliated Cancer Hospital, Guangxi Medical University, 71 Hedi Road, Nanning, Guangxi Zhuang Autonomous Region 530021, P.R. China

E-mail: tourtair@163.com

Dr Wei Tang, Department of Breast Surgery, Affiliated Cancer Hospital, Guangxi Medical University, 71 Hedi Road, Nanning, Guangxi Zhuang Autonomous Region 530021, P.R. China

E-mail: 21805195@qq.com

${ }^{*}$ Contributed equally

Key words: hepatocellular carcinoma, HOTAIR, oncogene, progression, metastasis interaction, focal adhesion, pathways in cancer were annotated with the HOTAIR and coexpression genes. In summary, the present analysis indicates that HOTAIR might be an oncogene in HCC. It functions though promoting tumor cell growth and inhibiting apoptosis. HOTAIR may potentially be involved in HCC metastatic progression by several pathways correlated to cell adhesion, and may be a therapeutic target in future.

\section{Introduction}

Hepatocellular carcinoma (HCC) is a commonly diagnosed cancer worldwide, with $\sim 782,500$ newly diagnosed cases and $>700,000$ HCC-related deaths in 2012 (1). Therapeutic strategies have been improved greatly including liver surgical resection, liver transplantation, chemotherapy and other modalities for HCC (2). However, the prognosis remains extremely dismal following surgical resection or liver transplantation in HCC patients, of which a long-term 5-year survival was reported ranging from $25-39 \%$, and a 5-year recurrence rate for whom had surgical treatment was reported ranging from $65-80 \%$ (3-5). Identification of a reliable oncogene is essential to predict the propensity of metastasis, prognosis and suggest further therapeutic decisions. Besides, integrated assessment for the underlying mechanisms has potential to identify novel therapeutic targets.

Long noncoding RNAs (lncRNAs) with >200 nucleotides are transcripts non-coding for proteins. LncRNAs have recently been found to involve in the development and progression of various kinds of cancer as key regulators of multiple biological processes (6). Typically, the lncRNA HOX transcript antisense intergenic RNA (HOTAIR) has been reported as a potential prognostic biomarker for cancers since it was discovered in 2007 (7). HOTAIR as a long and polyadenylated RNA that does not code for protein, and is expressed from the development HOXC locus located on chromosome 12q13.13 (7). HOTAIR overexpression has been extensively observed in multiple cancers and is reported to predict poor prognosis in esophageal carcinoma, gastric cancer, colorectal cancer, $\mathrm{HCC}$, breast cancer and other types of cancer $(8,9)$. There have been plenty of investigations carried out to study the potential molecular mechanisms of HOTAIR in cancer progression. Likewise, efforts were made to evaluate the expression 
pattern of HOTAIR in HCC and research its clinical implications $(10,11)$. However, the sample size ranged from 60 to 64 HCC patients and clinicopathological parameters of each individual previous study were relatively limited. The absence of bioinformatics analysis of the previous studies might have missed essential information for functional evaluation.

Therefore, the current study incorporating a larger HCC sample size, comprehensive clinicopathological parameters, and combined analyses of in vitro assays and functional assessments was designed to: 1) Evaluate the expression of HOTAIR in HCC tissues and adjacent non-tumor tissues, 2) evaluate the association between HOTAIR expression and clinical, histological, pathological and other biological features in HCC patients, 3) test the effects of HOTAIR on HCC using liver cancer cell lines, 4) find out genes associated or coordinated with HOTAIR and enrich in pathways.

\section{Materials and methods}

Patients. Eligible HCC participants were enrolled from the First Affiliated Hospital of Guangxi Medical University (Nanning, China) from March 2010 to December 2011. All participated samples were pathologically diagnosed with HCC by two independent pathologists. In brief, the clinicopathological parameters were collected and summarized in Table I, including age, gender, differentiation, tumor size, tumor nodes, clinical TNM stages, portal vein tumor embolus, metastasis, capsular infiltration and cirrhosis, vasoinvasion as well as other biomarkers, such as serum alpha fetal protein level detected by ELISA, nm23, P53, P21, vascular endothelial growth factor, microvessel density stained by CD34 through immunohistochemistry. The Ethical Committee of First Affiliated Hospital, Guangxi Medical University (Nanning, China) approved the current study. Informed consents were obtained from all the enrolled patients. Related research procedure was conducted based on the Helsinki Declaration.

Reverse transcription-quantitative polymerase chain reaction (RT-qPCR) analysis of HOTAIR expression levels. RNA isolation and RNA normalization were performed by the method descried in our previous reports $(12,13)$. Extracted RNA was analyzed by RT-qPCR using Applied Biosystems PCR7900 (Applied Biosystems; Thermo Fisher Scientific, Inc., Waltham, MA, USA). The expression of HOTAIR was assessed by reverse transcription RT-qPCR kits (Qiagen $\mathrm{GmbH}$, Hilden, Germany) based on the instructions described previously $(14,15)$. Human GAPDH was selected as the housekeeping reference for HOTAIR expression analysis. Primer sequences used in the PCR were as follows: HOTAIR 5'-GAG GGAGCCCAGAGTTACAGA-3' (sense), 5'-TCCTCCATT TCAGCCTTTCT-3' (antisense) and GAPDH: 5'-TGACTT CAACAGCGACACCCA-3' (sense), 5'-CACCCTGTTGCT GTAGCCAAA-3' (antisense). The HOTAIR expression was calculated with the formula $2^{-\Delta \Delta C q}(16)$.

Cell line and culture. The liver cancer cell line SMMC-7221 was purchased from Shanghai Institute of Cell Biology (Shanghai, China). The cell line was cultured at $37^{\circ} \mathrm{C}$ in $5 \%$ carbon dioxide in a humidified incubator following the recommended culture conditions $(14,15)$.
Lentiviral infection and gene transfection. Lentivirus containing HOTAIR shRNA segments (HOTAIR shRNA sequence was 5'-GAACGGGAGUACAGAGAGAUU-3') was purchased from Shanghai GenePharma Co., Ltd. (Shanghai, China). SMMC-7721 cells were infected with the viral suspension. HOTAIR 3' domain (nucleotides 1 to 300 of HOTAIR) and $5^{\prime}$ domain (nucleotides 1500 to 2146 of HOTAIR) were inserted into pcDNA3.1 (+) plasmid. pcDNA3.1 (+)-3' domain and pcDNA3.1 (+)-5' domain plasmids were transfected using Lipofectamine 2000 (Invitrogen; Thermo Fisher Scientific, Inc.) according to the manufacturer's protocols. The authors collected the transfected cell samples at 24 and 48 h points following transfection.

Analysis of cell line. After the SMMC-7721 cells were transfected with HOTAIR shRNA stably, the following steps to investigate the role and molecular mechanisms of HOTAIR in HCC progression were conducted at different time points (24 and $48 \mathrm{~h}$ ).

i) Cell viability was evaluated using fluorimetric detection of resorufin according to the manufacturer's protocols (CellTiter-Blue Cell Viability Assay, G8080, Promega Corporation, Madison, WI, USA). FL600 fluorescence plate reader was used for fluorimetry (ex: $560 \mathrm{~nm} / \mathrm{em}$ : $590 \mathrm{~nm}$; Bio-Tek Instruments, Inc., Winooski, VT, USA).

ii) Cell growth and apoptosis were evaluated with Hoechst 33342 (Sigma-Aldrich; Merck KGaA, Darmstadt, Germany) and propidium iodide (Sigma-Aldrich; Merck KGaA) double fluorescent chromatin staining. The number of cells in different states (necrotic, apoptotic and viable) were counted under x200 magnification, and each type of cell was calculated in 10 diverse fields in each well. The mean values compared with the mock control group were shown as the final results.

iii) A microplate reader was used to measure the cell proliferation ability (Scientific Multiskan MK3, Thermo Fisher Scientific, Inc.) at $490 \mathrm{~nm}$ after the liver cancer cells were processed by colorimetric tetrazolium (MTS) assay based on instruction (CellTiter96 Aqueous One Solution Cell Proliferation Assay G3580, Promega Corporation).

iv) The SMMC-7221 cells were plated into 96-well plate and treated as indicated. The cell lysates were harvested for caspase activity assays with caspase-Glo3/7 reagents (Promega Corporation). The luminescence of each sample was measured with a FL600 fluorescence plate-reading luminometer.

Construction of HOTAIR co-expression network and biological function analysis. HOTAIR coexpression genes were calculated with EBcoexpress package of R (17). The gene network map was drawn in Cytoscape Web (18), thereby providing figures to visually understand the relationship among coexpression genes. To clarify the functions of the HOTAIR and coexpression genes, the authors used the Kyoto Encyclopedia of Genes and Genomes (KEGG) pathway (http://www.genome.ad.jp/kegg/) and Gene Ontology (GO) (http://www.geneontology.org) analysis. GO can assign genes into hierarchical categories based on the associated aspects: Biological process, cellular component and molecular function (19). $\mathrm{P}<0.05$ was considered to indicate a statistically significant difference and was set as the threshold in GO and KEGG analyses. 
Table I. Relationship between the expression of HOTAIR and clinicopathological features in HCC.

Clinicopathological

features

Tissue

Adjacent liver

$\mathrm{HCC}$

Age

$<50$

$\geq 50$

Gender

Male

Female

Differentiation

High

Moderate

Low

Size

$<5 \mathrm{~cm}$

$\geq 5 \mathrm{~cm}$

Tumor nodes

Single

Multiple

Metastasis

No

Yes

Clinical TNM stage

I II

III IV

Portal vein tumor embolus

No

Yes

Vasoinvasion

No

Yes

Tumor capsular infiltration

No

Yes

HBV

-

$+$

AFP

-

$+$

Cirrhosis

No

Yes

NM23

$+$

Relative HOTAIR expression

$\mathrm{n}$ (patients)

Mean \pm standard deviation

$t$

P-value

95

95

46

49

75

20

6

60

29

18

77

52

43

46

49

22

73

63

32

59

36

45

50

17

78

41

38

50

45

20

75
$4.7600 \pm 1.78941$

$6.3618 \pm 1.99692$

$6.1169 \pm 1.99293$

$6.6226 \pm 1.98960$

$6.3641 \pm 1.96163$

$6.3530 \pm 2.17753$

$7.1333 \pm 1.58072$

$6.4928 \pm 2.19732$

$5.9310 \pm 1.55267$

$6.3317 \pm 2.17744$

$6.3688 \pm 1.96758$

$6.0300 \pm 1.84271$

$6.7630 \pm 2.12144$

$5.8957 \pm 1.88997$

$6.7994 \pm 2.01415$

$5.4091 \pm 1.16452$

$6.6489 \pm 2.10944$

$6.0397 \pm 1.72295$

$6.9959 \pm 2.35131$

$6.0231 \pm 1.69528$

$6.9169 \pm 2.33170$

$5.8644 \pm 1.73548$

$6.8094 \pm 2.12455$

$7.0106 \pm 1.83662$

$6.2204 \pm 2.01345$

$6.2466 \pm 2.16253$

$6.4779 \pm 2.01384$

$6.6014 \pm 1.97817$

$-1.236$

0.219

$6.0956 \pm 2.00590$

$5.5600 \pm 1.10043$

$6.5756 \pm 2.12943$ $5.823^{\mathrm{a}}$

$<0.001$

1.237

0.219

0.022

0.982

$F=1.259^{b}$

0.289

0.071

0.944

$-1.805$

0.075

$-2.252$

0.027

$-3.541$

0.001

$-2.039$

0.047

$-2.000$

0.050

$-2.358$

0.020

1.488

0.140

0.491

0.625 
Table I. Continued.

Relative HOTAIR expression

Clinicopathological

$\mathrm{n}$ (patients)

Mean \pm standard deviation

$t$

P-value

\section{P53}

$-$

$+$

P21

$-$

$+$

VEGF

-

$+$

Ki-67

Low

High

MVD

Low

High
40

55

62

33

25

70

47

48

47

48
$6.3548 \pm 1.91948$

$6.3669 \pm 2.06894$

$6.3227 \pm 1.92007$

$6.4352 \pm 2.16279$

$5.8720 \pm 1.74464$

$6.5367 \pm 2.06297$

$6.2809 \pm 1.94552$

$6.4410 \pm 2.06347$

$6.2894 \pm 2.21763$

$6.4327 \pm 1.77532$
$-0.029$

0.977

$-0.260$

0.795

$-1.437$

0.154

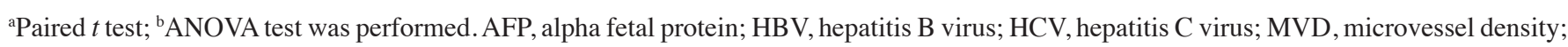
SD, standard deviation; TNM, tumor node metastasis; VEGF, vascular endothelial growth factor; HCC, hepatocellular carcinoma; MTDH, expression of metadherin; HOTAIR, HOX transcript antisense intergenic RNA.
Statistical analysis. SPSS software (version, 20.0; IBM SPSS, Armonk, NY, USA) was used for statistical analyses. For the analysis of the significance of two groups, the authors performed Student's test. Accordingly, they also applied one-way analysis of variance followed by Dunnett's post hoc test to analysis data from experiments in vitro. The associations between HOTAIR expression and clinicopathological parameters were tested using the Spearman rank correlation. The value of HOTAIR for differentiating the $\mathrm{HCC}$ from noncancerous tissues was tested by receiver operating characteristic (ROC) curve. All tests were two-sided, and $\mathrm{P}<0.05$ was considered to indicate a statistically significant difference.

\section{Results}

HOTAIR expression in HCC. A total of 95 eligible patients with diagnosed HCC, available cancer and adjacent non-cancer tissues were finally enrolled in the study. The age of the included individuals ranged from 29 to 82 years old, and the mean age was 52 years. HOTAIR expression was evaluated in 95 paired FFPE HCC and adjacent non-tumor tissues using RT-qPCR. The HOTAIR expression was significantly higher in HCC tissues, compared with the adjacent non-tumor tissue (at least $2 \mathrm{~cm}$ away from the tumor node; Fig. 1). All the clinicopathological parameters are presented in Table I.

HOTAIR expression and clinicopathological features of HCC. The relative expression of HOTAIR was $6.7994 \pm 2.01415$ in tissues with distant metastasis, significantly higher than those without distant metastasis $(5.8957 \pm 1.88997, \mathrm{P}=0.027)$. In patients with advanced (III-IV) stage, higher HOTAIR

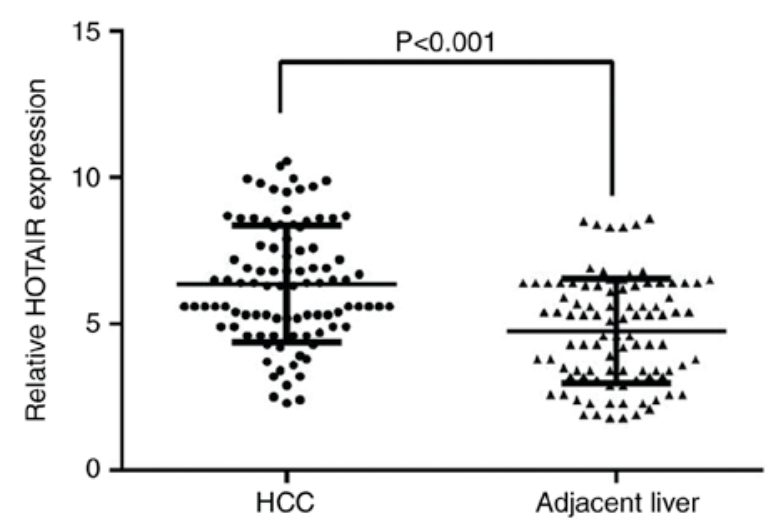

Figure 1. HOTAIR expression in HCC tissue and adjacent non-cancer tissues. HOTAIR, HOX transcript antisense intergenic RNA; HCC, hepatocellular carcinoma.

expression $(6.6489 \pm 2.10944)$ was observed than patients in early (I II) stage $(5.4091 \pm 1.16452, \mathrm{P}=0.001)$. Compared with those without portal vein tumor embolus $(6.0397 \pm 1.72295)$, the expression level of HOTAIR was obviously higher in patients with portal vein tumor embolus $(6.9959 \pm 2.35131, \mathrm{P}=0.047)$. Similarly, HOTAIR expression was remarkably higher in patients with vasoinvasion $(6.9169 \pm 2.33170)$ than those without vasoinvasion $(6.0231 \pm 1.69528, \mathrm{P}=0.005)$. In addition, the level of HOTAIR distinctly upregulated in patients with tumor capsular infiltration $(6.8094 \pm 2.12455)$ than those with complete liver capsule $(5.8644 \pm 1.73548, \mathrm{P}=0.020)$. In addition, HOTAIR expression was visibly higher in positive $\mathrm{nm} 23$ expression group of patients $(6.5756 \pm 2.12943)$ than 

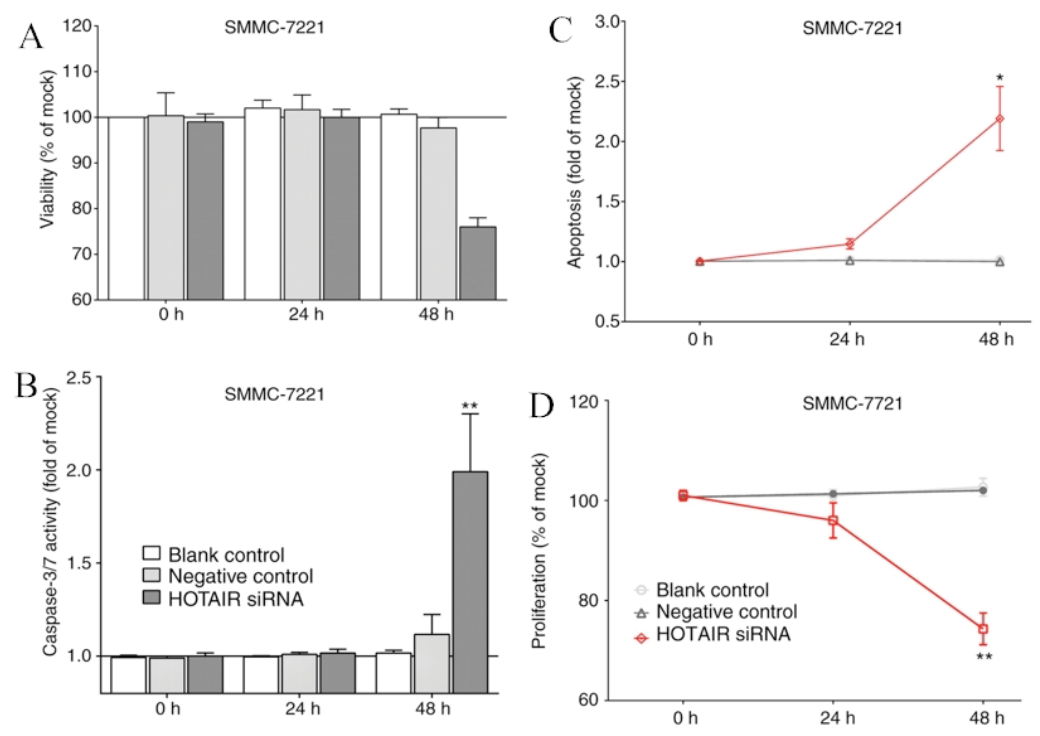

Figure 2. In vitro assays of SMMC-7221 cells. (A) Viability, (B) caspase-3/7 activity, (C) apoptosis and (D) proliferation. (C-D) Line of blank control almost overlapped with line of negative control. Points were calculated by taking the average of three independent experiments (mean \pm standard deviation). ${ }^{*} \mathrm{P}<0.05$, ${ }^{* *} \mathrm{P}<0.01$ and ${ }^{* * *} \mathrm{P}<0.001$ vs. blank and negative controls at the same time point. HOTAIR, HOX transcript antisense intergenic RNA; siRNA, small interfering RNA.

negative $\mathrm{nm} 23$ expression group $(5.5600 \pm 1.10043, \mathrm{P}=0.005)$. In Spearman analysis, the current result indicated that the relative expression of HOTAIR was significantly positive correlated with distant metastasis $(\mathrm{r}=0.210, \mathrm{P}=0.041)$, clinical TNM stage $(r=0.295, P=0.004)$, tumor capsular infiltration $(\mathrm{r}=0.235, \mathrm{P}=0.022)$ and $\mathrm{nm} 23(\mathrm{r}=0.228, \mathrm{P}=0.027)$. Nevertheless, no association was identified between HOTAIR expression and others clinicopathological features $(\mathrm{P}>0.05)$, including age, gender, differentiation, size and other parameters (Table I). Collectively, the results above demonstrated that the expression level of HOTAIR was associated with specific clinicopathological features that related to tumor deterioration and HOTAIR may play a vital role in promoting HCC progression.

Diagnostic significance of HOTAIR in HCC. The authors took advantage of ROC analysis to calculate the potential value of HOTAIR for diagnosing HCC. ROC analysis demonstrated that the Area Under The Curve (AUC) of HOTAIR was 0.715 [95\% confidence interval (CI): $0.643-0.787$ ] with a sensitivity of $45.3 \%$ and a specificity of $86.3 \%$ in distinguishing HCC and the cut off was 6.45 . The ROC analysis of HOTAIR and clinicopathological features revealed that HOTAIR levels remarkably discriminated HCC patients with distant metastasis, advanced TNM stage, tumor capsular infiltration and positive nm23 expression and the AUC was 0.621 (95\%CI: 0.509-0.734, sensitivity $=24.5 \%$, specificity $=97.8 \%), 0.701$ (95\%CI: $0.595-0.808$, sensitivity $=58.9 \%$, specificity $=81.8 \%), 0.636(95 \% \mathrm{CI}$ : $0.524-0.747$, sensitivity $=74.0 \%$, specificity $=51.1 \%$ ) and 0.661 $(95 \%$ CI:0.552-0.770, sensitivity $=49.3 \%$, specificity $=90.0 \%)$, respectively.

Expression of HOTAIR in the cell line and transfected cells. The behavior alterations of SMMC-7221cells on viability, proliferation, caspase-3/7 and apoptosis are presented in Fig. 2. The viability and proliferation of HOTAIR siRNA cells were significantly inhibited compared to that in control cells in $48 \mathrm{~h}$. Both levels of the caspase $3 / 7$ activity and apoptosis in the transfected cells exhibited higher than those in the control cells. The efficacy of the apoptosis measurement with SMMC-7221 cell line is presented in Fig. 3.

GO and KEGG pathway annotation. A total of 150 genes were identified in the coexpression analysis. The coexpression genes were then annotated through GO and KEGG pathway analyses. GO can organize genes into hierarchical categories and show the gene functions based on regulatory network of biological process, cellular component and molecular functions (Table II) (19). The genes showed dominant enrichments in the KEGG pathways of ECM-receptor interaction, focal adhesion and pathways in cancer (Table III).

\section{Discussion}

HCC is a critical health problem worldwide and is an aggressive disease. The high morbidity and mortality rates of this malignance are not arbitrary but raise the researcher's attention. In the present study, the HOTAIR expression level was detected significantly higher in the HCC tissues than the adjacent non-tumor tissues. The HOTAIR expression levels were observed significantly higher in tumor samples from patients with distant metastasis, advanced stage, portal vein tumor embolus, vasoinvasion, tumor capsular infiltration or positive $\mathrm{nm} 23$ than those from patients without distant metastasis, advanced stage, portal vein tumor embolus, vasoinvasion, tumor capsular infiltration or positive $\mathrm{nm} 23$ correspondingly. In vitro assays, the silencing of HOTAIR in liver cancer cells induced the promotion of apoptosis and inhibition of cell proliferation. ECM-receptor interaction, focal adhesion and pathways in cancer were annotated with the HOTAIR and coexpression genes.

HOTAIR has been evaluated extensively within the context of cancer prognosis (20). A meta-analysis for patients with solid 


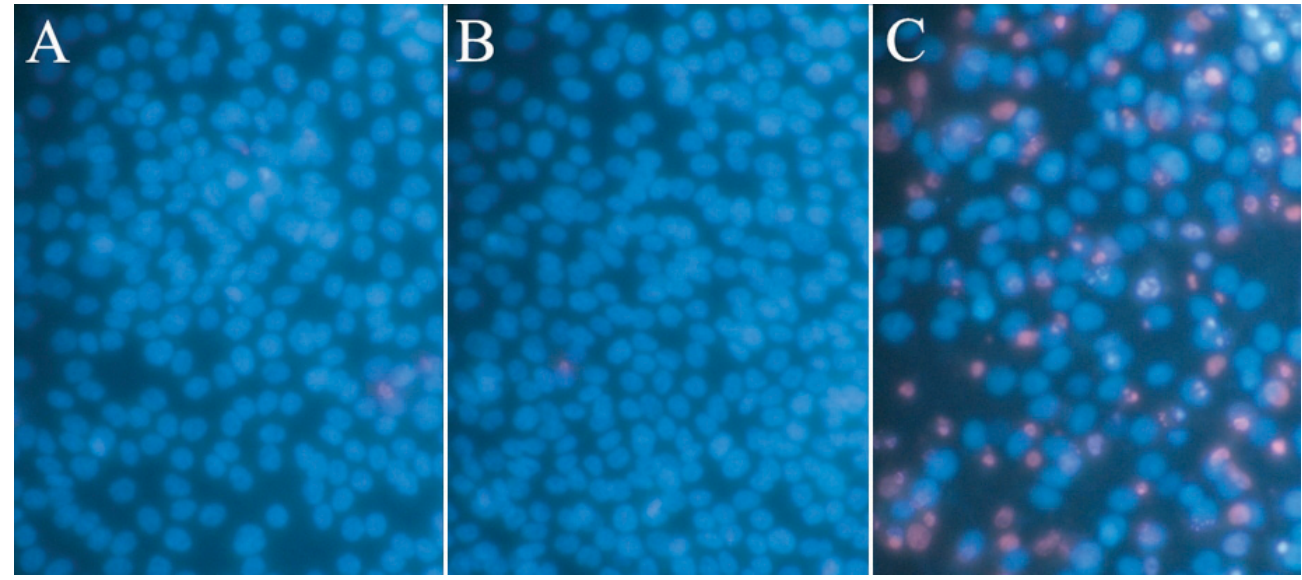

Figure 3. The efficacy of the apoptosis was measured with propidium iodide double fluorescent chromatin staining (magnification, $\mathrm{x} 200$ ). (A) $48 \mathrm{~h}$ SMMC-7221 Blank control; (B) 48 h SMMC-7221 negative control; (C) 48 h SMMC-7221 HOTAIR siRNA. HOTAIR, HOX transcript antisense intergenic RNA; siRNA, small interfering RNA.

tumors reported that higher HOTAIR expression could significantly predict worse overall survival (21). In gastric cancer, high expression of HOTAIR was significantly associated with the depth of tumor invasion, lymph node metastasis, vessel invasion, lymphatic vessel involvement and TNM stage (22). The incidence of lymph node metastasis was illustrated to be higher in cancer patients with high HOTAIR expression compared to patients with low HOTAIR expression (23). Likewise, HOTAIR was indicated to be a potential predictor of poor relapse-free survival, metastasis-free survival and disease-free survival in cervical, ovarian, breast and endometrial cancer patients (24).

To the best of the authors' knowledge, the current study contained a larger HCC sample than the previous investigations for HCC and HOTAIR. Compared with adjacent non-HCC tissue, the analysis yielded that HOTAIR expressed higher in the HCC tissue, which was observed in several previous reports, implying the diagnosis value of HOTAIR in HCC patients (11,25-27). Ishibashi et al (11) found that patients with HOTAIR expression had significantly larger primary tumor size than those without HOTAIR expression. A vast majority of samples from study of Geng et al (26) showed higher levels of HOTAIR in tumor tissues than in adjacent non-tumor tissues, and HOTAIR expression were significantly higher in tumor tissues from patients with lymph node metastasis. The HOTAIR expression was reported increased at least two-fold in HCC tumor tissues relative to that in non-tumor tissues (27). These evidences were robust to illustrate that HOTAIR might be an oncogene for HCC.

In the current study, a Spearman's rank correlation coefficient analysis showed that the higher HOTAIR expression level was significantly correlated with metastasis, advanced TNM stage and portal vein tumor embolus. Besides, HOTAIR expression was remarkably higher in patients with vasoinvasion than those without vasoinvasion, as well as in capsular infiltration than those with complete liver capsule. The clinicopathological characteristics of vasoinvasion, capsular infiltration always correlated to invasion and progression, suggesting HOTAIR might be involved in HCC invasion and serve as poor prognosis predictor. These findings allowed HOTAIR to be a potential molecular for risk classification of HCC patients, and that molecular-based tumor risk stratification was important for the decision of individual diagnosis and therapy $(25,28)$. ROC analysis demonstrated that the AUC of HOTAIR was 0.715 (95\%CI: 0.643-0.787) with a sensitivity of $45.3 \%$ and a specificity of $86.3 \%$ in distinguishing HCC. The authors speculated that an individual biomarker was insufficient to diagnosis malignant disease, and a combined analysis of the well-studied diagnosis biomarkers might provide novel insight into the diagnosis method for HCC.

Given the clinical implications of HOTAIR upon HCC and the currently poor interpretation of its mechanisms, a series of in vitro assays were conducted to uncover the effects of HOTAIR on HCC. After silencing HOTAIR expression, the proliferation and viability of SMMC-7221 cells were depressed, while the significant promotion of apoptosis and caspase ability was observed. Furthermore, the level of the depressed and promoted effects was associated with the transfection processing time, indicating the high HOTAIR expression level was related to the increased HCC progression risk. Taken the in vitro results together, it demonstrated that HOTAIR involved in promoting HCC cell growth and inhibiting apoptosis, explaining the phenomenon that the increased HOTAIR expression was found in HCC tissues and the significant associations with the advanced TNM stage (26). Moreover, the ROC analysis of HOTAIR and distant metastasis, advanced TNM stage and tumor capsular infiltration also indicated expression of HOTAIR might be used for molecular based tumor staging or progressive risk stratification. Since increased HOTAIR expression was not exclusively specific in one particular cancer, and observed promoting cell proliferation, invasion and migration in variant types of tumor cells, the authors speculated high HOTAIR expression could be a potential marker for predicting poor prognosis in multiple types of cancers $(20,29,30)$.

It was well recognized that HOTAIR interacted with multiple genes in a manner dependent on polycomb repressive complex 2 (PRC2), which could induce histone H3 lysine 27 (H3K27) methylation and thus silence transcription of the HOXD locus, and eventually cause epigenetic silencing of metastasis suppressor genes (31-33). Knockdown of HOTAIR was associated with reductions in levels of vascular endothelial growth factor protein and matrix metalloproteinase-9, which were vital for cell motility and metastasis (26). Besides, 
Table II. Gene ontology terms enriched in HOTAIR and coexpression genes $(\mathrm{P}<0.05, \mathrm{FDR}<0.1)$.

\begin{tabular}{|c|c|c|c|}
\hline GO analysis & Count & P-value & FDR \\
\hline \multicolumn{4}{|l|}{ Biological process } \\
\hline GO:0001501 skeletal system development & 26 & $2.78 \times 10^{18}$ & $4.37 \times 10^{15}$ \\
\hline GO:0048598 embryonic morphogenesis & 20 & $3.54 \times 10^{12}$ & $5.57 \times 10^{9}$ \\
\hline GO:0048705 skeletal system morphogenesis & 13 & $8.17 \times 10^{11}$ & $1.29 \times 10^{7}$ \\
\hline GO:0048706 embryonic skeletal system development & 11 & $4.84 \times 10^{10}$ & $7.61 \times 10^{7}$ \\
\hline GO:0003002 regionalization & 14 & $5.47 \times 10^{9}$ & $8.60 \times 10^{6}$ \\
\hline GO:0009952 anterior/posterior pattern formation & 12 & $1.44 \times 10^{8}$ & $2.27 \times 10^{5}$ \\
\hline GO:0007389 pattern specification process & 15 & $2.58 \times 10^{8}$ & $4.07 \times 10^{5}$ \\
\hline GO:0030326 embryonic limb morphogenesis & 10 & $2.87 \times 10^{8}$ & $4.52 \times 10^{5}$ \\
\hline GO:0035113 embryonic appendage morphogenesis & 10 & $2.87 \times 10^{8}$ & $4.52 \times 10^{5}$ \\
\hline GO:0035108 limb morphogenesis & 10 & $8.95 \times 10^{8}$ & $1.41 \times 10^{4}$ \\
\hline GO:0035107 appendage morphogenesis & 10 & $8.95 \times 10^{8}$ & $1.41 \times 10^{4}$ \\
\hline GO:0060173 limb development & 10 & $1.26 \times 10^{7}$ & $1.99 \times 10^{4}$ \\
\hline GO:0048736 appendage development & 10 & $1.26 \times 10^{7}$ & $1.99 \times 10^{4}$ \\
\hline GO:0048704 embryonic skeletal system morphogenesis & 8 & $3.23 \times 10^{7}$ & $5.09 \times 10^{4}$ \\
\hline GO:0006355 regulation of transcription, DNA-dependent & 35 & $5.25 \times 10^{7}$ & $8.26 \times 10^{4}$ \\
\hline GO:0051252 regulation of RNA metabolic process & 35 & $8.87 \times 10^{7}$ & $1.40 \times 10^{3}$ \\
\hline GO:0048562 embryonic organ morphogenesis & 10 & $1.12 \times 10^{6}$ & $1.77 \times 10^{3}$ \\
\hline GO:0048568 embryonic organ development & 11 & $1.12 \times 10^{6}$ & $1.77 \times 10^{3}$ \\
\hline GO:0043009 chordate embryonic development & 14 & $2.27 \times 10^{6}$ & $3.57 \times 10^{3}$ \\
\hline GO:0009792 embryonic development ending in birth or egg hatching & 14 & $2.50 \times 10^{6}$ & $3.94 \times 10^{3}$ \\
\hline GO:0006357 regulation of transcription from RNA polymerase II promoter & 20 & $4.27 \times 10^{6}$ & $6.73 \times 10^{3}$ \\
\hline GO:0060348 bone development & 9 & $6.12 \times 10^{06}$ & $9.64 \times 10^{3}$ \\
\hline GO:0045893 positive regulation of transcription, DNA-dependent & 15 & $2.55 \times 10^{5}$ & $4.01 \times 10^{2}$ \\
\hline GO:0051216 cartilage development & 7 & $2.68 \times 10^{5}$ & $4.23 \times 10^{2}$ \\
\hline GO:0051254 positive regulation of RNA metabolic process & 15 & $2.79 \times 10^{5}$ & $4.40 \times 10^{2}$ \\
\hline GO:0031328 positive regulation of cellular biosynthetic process & 18 & $2.84 \times 10^{5}$ & $4.46 \times 10^{2}$ \\
\hline GO:0009954 proximal/distal pattern formation & 5 & $3.03 \times 10^{5}$ & $4.76 \times 10^{2}$ \\
\hline GO:0009891 positive regulation of biosynthetic process & 18 & $3.41 \times 10^{5}$ & $5.36 \times 10^{2}$ \\
\hline GO:0001503 ossification & 8 & $3.67 \times 10^{5}$ & $5.77 \times 10^{2}$ \\
\hline GO:0007155 cell adhesion & 18 & $3.73 \times 10^{5}$ & $5.87 \times 10^{2}$ \\
\hline GO:0022610 biological adhesion & 18 & $3.80 \times 10^{5}$ & $5.98 \times 10^{2}$ \\
\hline GO:0045944 positive regulation of transcription from RNA polymerase II promoter & 13 & $3.95 \times 10^{5}$ & $6.22 \times 10^{2}$ \\
\hline GO:0045941 positive regulation of transcription & 16 & $4.02 \times 10^{5}$ & $6.33 \times 10^{2}$ \\
\hline GO:0021515 cell differentiation in spinal cord & 5 & $4.27 \times 10^{5}$ & $6.72 \times 10^{2}$ \\
\hline GO:0051173 positive regulation of nitrogen compound metabolic process & 17 & $4.94 \times 10^{5}$ & $7.78 \times 10^{2}$ \\
\hline GO:0010628 positive regulation of gene expression & 16 & $5.64 \times 10^{5}$ & $8.88 \times 10^{2}$ \\
\hline GO:0010557 positive regulation of macromolecule biosynthetic process & 17 & $5.94 \times 10^{5}$ & $9.34 \times 10^{2}$ \\
\hline \multicolumn{4}{|l|}{ Cellular component } \\
\hline GO:0031012 extracellular matrix & 18 & $4.61 \times 10^{10}$ & $5.51 \times 10^{7}$ \\
\hline GO:0005578 proteinaceous extracellular matrix & 16 & $1.07 \times 10^{8}$ & $1.28 \times 10^{5}$ \\
\hline GO:0044420 extracellular matrix part & 10 & $1.83 \times 10^{7}$ & $2.20 \times 10^{4}$ \\
\hline GO:0044421 extracellular region part & 22 & $4.77 \times 10^{6}$ & $5.713 \times 10^{3}$ \\
\hline \multicolumn{4}{|l|}{ Molecular function } \\
\hline GO:0043565 sequence-specific DNA binding & 25 & $5.21 \times 10^{11}$ & $6.68 \times 10^{8}$ \\
\hline GO:0003700 transcription factor activity & 29 & $1.59 \times 10^{9}$ & $2.04 \times 10^{6}$ \\
\hline GO:0030528 transcription regulator activity & 33 & $1.36 \times 10^{7}$ & $1.74 \times 10^{4}$ \\
\hline GO:0005201 extracellular matrix structural constituent & 7 & $6.40 \times 10^{5}$ & $8.21 \times 10^{2}$ \\
\hline
\end{tabular}

FDR, False Discovery Rate; HOTAIR, HOX transcript antisense intergenic RNA. 
Table III. KEGG pathways enriched in HOTAIR and coexpression genes $(\mathrm{P}<0.05)$.

\begin{tabular}{lcccl}
\hline Pathway & Count & P-value & FDR & Genes \\
\hline KEGG & & & & \\
hsa04512:ECM-receptor interaction & 5 & $7.85 \times 10^{4}$ & $7.28 \times 10^{1}$ & TNC, COL1A2, COL6A1, LAMB1, COL5A2 \\
hsa04510:Focal adhesion & 5 & $1.79 \times 10^{2}$ & $1.55 \times 10^{1}$ & TNC, COL1A2, COL6A1, LAMB1, COL5A2 \\
hsa05200:Pathways in cancer & 6 & $2.32 \times 10^{2}$ & $1.96 \times 10^{1}$ & FGFR1, HDAC2, TGFB3, LAMB1, GLI3, MMP2
\end{tabular}

KEGG, Kyoto Encyclopedia of Genes and Genomes; FDR, False Discovery Rate; HOTAIR, HOX transcript antisense intergenic RNA.

HOTAIR was found to promote cell migration and invasion via inhibiting RNA binding motif protein 38 (RBM38) in HCC cells, since knockdown of HOTAIR raised the expression RBM38 both on mRNA levels and protein levels, and the increased expression of RBM38 could restrain cell motility (10). A previous study reported HOTAIR enhanced cell viability and escaped G1-phase arrest through suppressing miRNA-218 expression and inhibiting P14 and P16 signaling. Suppressing oncogene Bmi-1 was shown to be a functional target of miR-218, and the main downstream targets signaling, P16(lnk4a) and P14(ARF), were inactivated in HOTAIR tumorigenesis (34). In HepG2 cells, the phenomenon that microRNA miR-125a-5p decreased and released caspase 2 to promote HCC cell apoptosis was reported after HOTAIR knockdown (35). Therefore, HOTAIR might control HCC cell proliferation through interacting with microRNAs. The identification of microRNAs and target genes of HOTAIR in $\mathrm{HCC}$ is of great importance to understand HCC pathogenesis.

Although accumulating evidence has revealed substantial biological functions of HOTAIR, the precise mechanisms remained largely to be explored. Coexpression network analysis, which was used to find genes having similar or coordinated expression patterns, was carried out to identify HOTAIR coexpression genes $(36,37)$. GO enrichment analysis revealed that a majority of the HOTAIR and coexpression genes were involved in pathways related to cell adhesion, biological adhesion, transcription regulator activity, transcription factor activity and sequence-specific DNA binding. These data supported that most lncRNAs worked with DNA-binding proteins, and epigenetically regulated the expression of multiple genes $(7,31)$. It is noteworthy that in HCC patients with positive $\mathrm{nm} 23$, which has also been reported to contribute to metastasis (38), HOTAIR expression level was higher than that in nm23 negative patients. To some extent, the significant correlation between HOTAIR expression and nm23 status suggested that they might function in a manner of cooperation. However, a previous study reports that the absence of nm23 in HCC is significantly correlated with extrahepatic metastasis (39). In mice models, nm 23 has been also observed overexpressed in the HCC, whereas lung metastasis was found to be promoted in the transgenic nm23-null mice (40). The oncogenic and metastasis role of the nm23 is still debated. The contradiction between the results suggests future studies with larger samples are needed to illustrate the function of $\mathrm{nm} 23$.

In addition, three pathways were screened out using KEGG analysis for the HOTAIR and coexpression genes, such as ECM-receptor interaction, focal adhesion and pathways in cancer. Most of these pathways were reported involving in cancer progression (41). Typically, pathways linked to cell spread and migration was both annotated in GO and KEGG, such as cell adhesion, biological adhesion and focal adhesion $(42,43)$. It strongly suggests that HOTAIR might be a therapeutic target to decrease metastasis risk, which remained a field to be investigated (44).

Concerning with the limitations, the absence of survival data hindered this study to gain prognosis value of HOTAIR in HCC. As for control tissue origin, only the adjacent noncancerous tissues including cirrhotic and noncirrhotic liver tissues were available. Given the multi-stages hepatocarcinogenesis process of HCC (45), the authors suggested that the combined analysis of normal tissues might reveal more in-depth mechanisms of HOTAIR in HCC progression. A future study to validate HOTAIR mechanisms in HCC patients is necessary.

The current study suggested that HOTAIR was an oncogene in HCC. It functioned though promoting tumor cell growth and inhibiting apoptosis. HOTAIR potentially regulated HCC metastatic progression by several pathways correlated cell adhesion, and might be a therapeutic target in future.

\section{Acknowledgements}

The present study was supported by grants from the Fund of Future Academic Star of Guangxi Medical University (grant no. WLXSZX16001) and the Scientific Research Project of the Department of Education in Guangxi Zhuang Autonomous Region (grant nos. LX2014064 and 201204LX044).

\section{References}

1. Torre LA, Bray F, Siegel RL, Ferlay J, Lortet-Tieulent J and Jemal A: Global cancer statistics, 2012. CA Cancer J Clin 65: 87-108, 2015.

2. Kanda T, Ogasawara S, Chiba T, Haga Y, Omata M and Yokosuka O: Current management of patients with hepatocellular carcinoma. World J Hepatol 7: 1913-1920, 2015.

3. Kamiyama T, Nakanishi K, Yokoo H, Kamachi H, Tahara M, Suzuki T, Shimamura T, Furukawa H, Matsushita M and Todo S: Recurrence patterns after hepatectomy of hepatocellular carcinoma: Implication of Milan criteria utilization. Ann Surg Oncol 16: 1560-1571, 2009.

4. Sakon M, Umeshita K, Nagano H, Eguchi H, Kishimoto S, Miyamoto A, Ohshima S, Dono K, Nakamori S, Gotoh M and Monden M: Clinical significance of hepatic resection in hepatocellular carcinoma: Analysis by disease-free survival curves. Arch Surg 135: 1456-1459, 2000.

5. Portolani N, Coniglio A, Ghidoni S, Giovanelli M, Benetti A, Tiberio GA and Giulini SM: Early and late recurrence after liver resection for hepatocellular carcinoma: Prognostic and therapeutic implications. Ann Surg 243: 229-235, 2006. 
6. Kugel JF and Goodrich JA: Non-coding RNAs: Key regulators of mammalian transcription. Trends Biochem Sci 37: 144-151, 2012.

7. Rinn JL, Kertesz M, Wang JK, Squazzo SL, Xu X, Brugmann SA, Goodnough LH, Helms JA, Farnham PJ, Segal E and Chang HY: Functional demarcation of active and silent chromatin domains in human HOX loci by noncoding RNAs. Cell 129: 1311-1323, 2007.

8. Zhang S, Chen S, Yang G, Gu F, Li M, Zhong B, Hu J, Hoffman A and Chen M: Long noncoding RNA HOTAIR as an independent prognostic marker in cancer: A meta-analysis. PLoS One 26 e105538, 2014.

9. Deng Q, Sun H, He B, Pan Y, Gao T, Chen J, Ying H, Liu X, Wang F, Xu Y and Wang S: Prognostic value of long non-coding RNA HOTAIR in various cancers. PLoS One 9: e110059, 2014.

10. Ding C, Cheng S, Yang Z, Lv Z, Xiao H, Du C, Peng C, Xie H, Zhou L, Wu J and Zheng S: Long non-coding RNA HOTAIR promotes cell migration and invasion via down-regulation of RNA binding motif protein 38 in hepatocellular carcinoma cells. Int J Mol Sci 15: 4060-4076, 2014.

11. Ishibashi M, Kogo R, Shibata K, Sawada G, Takahashi Y, Kurashige J, Akiyoshi S, Sasaki S, Iwaya T, Sudo T, et al: Clinical significance of the expression of long non-coding RNA HOTAIR in primary hepatocellular carcinoma. Oncol Rep 29: 946-950, 2013

12. Chen G, Umelo IA, Lv S, Teugels E, Fostier K, Kronenberger P, Dewaele A, Sadones J, Geers C and De Grève J: miR-146a inhibits cell growth, cell migration and induces apoptosis in non-small cell lung cancer cells. PLoS One 8:e60317, 2013.

13. Rong M, He R, Dang Y and Chen G: Expression and clinicopathological significance of miR-146a in hepatocellular carcinoma tissues. Ups J Med Sci 119: 19-24, 2014.

14. Huang S, He R, Rong M, Dang Y and Chen G: Synergistic effect of MiR-146a mimic and cetuximab on hepatocellular carcinoma cells. Biomed Res Int 2014: 384121, 2014.

15. Dang YW, Zeng J, He RQ, Rong MH, Luo DZ and Chen G: Effects of miR-152 on cell growth inhibition, motility suppression and apoptosis induction in hepatocellular carcinoma cells. Asian Pac J Cancer Prev 15: 4969-4976, 2014.

16. Livak KJ and Schmittgen TD: Analysis of relative gene expression data using real-time quantitative PCR and the 2(-Delta Delta C(T)) method. Methods 25: 402-408, 2001.

17. Dawson JA, Ye S and Kendziorski C: R/EBcoexpress: An empirical Bayesian framework for discovering differential co-expression. Bioinformatics 28: 1939-1940, 2012.

18. Lopes CT, Franz M, Kazi F, Donaldson SL, Morris Q and Bader GD: Cytoscape web: An interactive web-based network browser. Bioinformatics 26: 2347-2348, 2010.

19. Ashburner M, Ball CA, Blake JA, Botstein D, Butler $H$ Cherry JM, Davis AP, Dolinski K, Dwight SS, Eppig JT, et al: Gene ontology: Tool for the unification of biology. The gene ontology consortium. Nat Genet 25: 25-29, 2000.

20. Serghiou S, Kyriakopoulou A and Ioannidis JP: Long noncoding RNAs as novel predictors of survival in human cancer: A systematic review and meta-analysis. Mol Cancer 15: 50, 2016.

21. Miao Z, Ding J, Chen B, Yang Y and Chen Y: HOTAIR overexpression correlated with worse survival in patients with solid tumors. Minerva Med 107: 392-400, 2016.

22. Liu FT, Qiu C, Luo HL, Zhang Y, Xia GF, Hao TF and Zhu PQ: The association of HOTAIR expression with clinicopathological features and prognosis in gastric cancer patients. Panminerva Med 58: 167-174, 2016.

23. Cai B, Wu Z, Liao K and Zhang S: Long noncoding RNA HOTAIR can serve as a common molecular marker for lymph node metastasis: A meta-analysis. Tumour Biol 35: 8445-8450, 2014.

24. Li J, Wen W, Zhao S, Wang J, Chen J, Wang Y and Zhang Q: Prognostic role of HOTAIR in four estrogen-dependent malignant tumors: A meta-analysis. Onco Targets Ther 8: 1471-1482, 2015.

25. Yang Z, Zhou L, Wu LM, Lai MC, Xie HY, Zhang F and Zheng SS: Overexpression of long non-coding RNA HOTAIR predicts tumor recurrence in hepatocellular carcinoma patients following liver transplantation. Ann Surg Oncol 18: 1243-1250, 2011.

26. Geng YJ, Xie SL, Li Q, Ma J and Wang GY: Large intervening non-coding RNA HOTAIR is associated with hepatocellular carcinoma progression. J Int Med Res 39: 2119-2128, 2011.
27. Gao JZ, Li J, Du JL and Li XL: Long non-coding RNA HOTAIR is a marker for hepatocellular carcinoma progression and tumor recurrence. Oncol Lett 11: 1791-1798, 2016.

28. Schmidt C and Marsh JW: Molecular signature for HCC: Role in predicting outcomes after liver transplant and selection for potential adjuvant treatment. Curr Opin Organ Transplant 15: 277-282, 2010.

29. Li X, Wu Z, Mei Q, Li X, Guo M, Fu X and Han W: Long non-coding RNA HOTAIR, a driver of malignancy, predicts negative prognosis and exhibits oncogenic activity in oesophageal squamous cell carcinoma. Br J Cancer 109: 2266-2278, 2013.

30. Jing L, Yuan W, Ruofan D, Jinjin Y and Haifeng Q: HOTAIR enhanced aggressive biological behaviors and induced radio-resistance via inhibiting p21 in cervical cancer. Tumour Biol 36: 3611-3619, 2015.

31. Gupta RA, Shah N, Wang KC, Kim J, Horlings HM, Wong DJ, Tsai MC, Hung T, Argani P, Rinn JL, et al: Long non-coding RNA HOTAIR reprograms chromatin state to promote cancer metastasis. Nature 464: 1071-1076, 2010.

32. Kogo R, Shimamura T, Mimori K, Kawahara K, Imoto S, Sudo T, Tanaka F, Shibata K, Suzuki A, Komune S, et al: Long noncoding RNA HOTAIR regulates polycomb-dependent chromatin modification and is associated with poor prognosis in colorectal cancers. Cancer Res 71: 6320-6326, 2011.

33. Tsai MC, Manor O, Wan Y, Mosammaparast N, Wang JK, Lan F, Shi Y, Segal E and Chang HY: Long noncoding RNA as modular scaffold of histone modification complexes. Science 329: 689-693, 2010.

34. Fu WM, Zhu X, Wang WM, Lu YF, Hu BG, Wang H, Liang WC, Wang SS, Ko CH, Waye MM, et al: Hotair mediates hepatocarcinogenesis through suppressing miRNA-218 expression and activating P14 and P16 signaling. J Hepatol 63: 886-895, 2015.

35. Tang L, Shen H, Li X, Li Z, Liu Z, Xu J, Ma S, Zhao X, Bai X, Li M, et al: MiR-125a-5p decreases after long non-coding RNA HOTAIR knockdown to promote cancer cell apoptosis by releasing caspase 2. Cell Death Dis 7: e2137, 2016.

36. Carlson MR, Zhang B, Fang Z, Mischel PS, Horvath S and Nelson SF: Gene connectivity, function, and sequence conservation: Predictions from modular yeast co-expression networks. BMC Genomics 7: 40, 2006.

37. Ma S, Shi M, Li Y, Yi D and Shia BC: Incorporating gene co-expression network in identification of cancer prognosis markers. BMC Bioinformatics 11: 271, 2010.

38. Guo H, Nan K, Hu T, Meng J, Hui W, Zhang X, Qin H and Sui C: Prognostic significance of co-expression of $\mathrm{nm} 23$ and $\mathrm{p} 57$ protein in hepatocellular carcinoma. Hepatol Res 40: 1107-1116, 2010.

39. An R, Meng J, Shi Q, Dai XX, Chen JH, Lei YJ, Shan B, Gao C, Chu YL and Dong XP: Expressions of nucleoside diphosphate kinase (nm23) in tumor tissues are related with metastasis and length of survival of patients with hepatocellular carcinoma. Biomed Environ Sci 23: 267-272, 2010.

40. Boissan M, Wendum D, Arnaud-Dabernat S, Munier A, Debray M, Lascu I, Daniel JY and Lacombe ML: Increased lung metastasis in transgenic NM23-Null/SV40 mice with hepatocellular carcinoma. J Natl Cancer Inst 97: 836-845, 2005.

41. Liu P, Jiang W, Ren H, Zhang $\mathrm{H}$ and Hao J: Exploring the molecular mechanism and biomakers of liver cancer based on gene expression microarray. Pathol Oncol Res 21: 1077-1083, 2015.

42. Kim DH and Wirtz D: Predicting how cells spread and migrate: Focal adhesion size does matter. Cell Adh Migr 7: 293-296, 2013

43. Yang CC, Chang SF, Chao JK, Lai YL, Chang WE, Hsu WH and Kuo WH: Activation of AMP-activated protein kinase attenuates hepatocellular carcinoma cell adhesion stimulated by adipokine resistin. BMC Cancer 14: 112, 2014.

44. $\mathrm{Li} \mathrm{CH}$ and Chen Y: Targeting long non-coding RNAs in cancers: Progress and prospects. Int J Biochem Cell Biol 45: 1895-1910, 2013.

45. Bugianesi E, Leone N, Vanni E, Marchesini G, Brunello F, Carucci P, Musso A, De Paolis P, Capussotti L, Salizzoni $M$ and Rizzetto $M$ : Expanding the natural history of nonalcoholic steatohepatitis: From cryptogenic cirrhosis to hepatocellular carcinoma. Gastroenterology 123: 134-140, 2002 . 